\title{
On the Poisson Integral of Step Functions and Minimal Surfaces
}

\author{
Allen Weitsman
}

Abstract. Applications of minimal surface methods are made to obtain information about univalent harmonic mappings. In the case where the mapping arises as the Poisson integral of a step function, lower bounds for the number of zeros of the dilatation are obtained in terms of the geometry of the image.

\section{Introduction}

Let $f$ be a univalent harmonic mapping of the unit disk $U$. By this it is meant not only that $f$ is $1-1$ and harmonic, but also that $f$ is sense preserving. Then $f$ can be written

$$
f=h+\bar{g}
$$

where $h$ and $g$ are analytic in $U$. If $a(\zeta)$ is defined by

$$
a(\zeta)=\overline{f_{\bar{\zeta}}(\zeta)} / f_{\zeta}(\zeta)=g^{\prime}(\zeta) / h^{\prime}(\zeta),
$$

then $a(\zeta)$ is analytic and $|a(\zeta)|<1$ in $U$. We shall refer to $a(\zeta)$ as the analytic dilatation of $f$. General function theoretic properties of univalent harmonic mappings may be found in [CS-S]. The case where $a(\zeta)$ is a finite Blaschke product is of special interest since this case arises in taking the Poisson integrals of step functions [S-S]. This connection has been studied in [HS] and [S-S]. In [W], a method was developed using the theory of minimal surfaces to study univalent harmonic mappings. We shall continue this study.

In Section 2 we shall review the definitions of the height function and conjugate height function introduced in [W], along with their relevant properties. In Section 3 we shall prove the comparison principle for the height function. In Section 4 we collect some results from the theory of minimal surfaces which enable us to use the conjugate height function as a combinatorial tool. In Section 5 we give some applications to the Poisson integrals of step functions.

\section{The Height Function and Conjugate Height Function}

Using the Weierstrass representation [O, p. 63], we associate with $f$ a minimal surface given parametrically in a simply connected subdomain $N \subseteq U$ where $a(\zeta)$ does not have a zero of odd order.

Received by the editors February 11, 2000.

AMS subject classification: 30C62, 31A05, 31A20, $49 \mathrm{Q} 05$.

Keywords: harmonic mappings, dilatation, minimal surfaces.

(C)Canadian Mathematical Society 2002. 
With $g$ and $h$ as in (1.1) we define up to an additive constant, a branch of

$$
F(\zeta)=2 i \int \sqrt{h^{\prime}(\zeta) g^{\prime}(\zeta)} d \zeta=2 i \int h^{\prime}(\zeta) \sqrt{a(\zeta)} d \zeta=2 i \int f_{\zeta}(\zeta) \sqrt{a(\zeta)} d \zeta
$$

Then, by (1.2) it follows that a branch of $F$ can be defined in $N$, and for $\zeta \in N$,

$$
\zeta \rightarrow(f(\zeta), \operatorname{Re} F(\zeta))
$$

gives a parametric representation of a minimal surface. Here we have identified $\mathbb{R}^{2}$ with $\mathbb{C}$ by $(x, y) \leftrightarrow(\operatorname{Re} f, \operatorname{Im} f)$.

Let $\hat{U}$ be the Riemann surface of the function $\sqrt{a(\zeta)}$. Then $\hat{U}$ has algebraic branch points corresponding to those points $\zeta \in U$ for which $a(\zeta)$ has a zero of odd order. Specifically, $\hat{U}$ can be concretely described (the analytic configuration [S, pp. 69-74]) in terms of function elements $\left(\alpha, F_{\alpha}\right)$ where $\alpha \in U$, and $F_{\alpha}$ is a power series expansion of a branch of $F$ in a neighborhood of $\alpha$ if $a(\zeta)$ does not have a zero of odd order at $\zeta=\alpha$, and $F_{\alpha}$ a power series in $\sqrt{\zeta-\alpha}$ otherwise. The mapping $p:\left(\alpha, F_{\alpha}\right) \rightarrow \alpha$ is the projection of the surface so realized. The mapping $F$ may now be lifted to a mapping $\hat{F}$ on $\hat{U}$.

By continuation, we may induce a mapping $\hat{U} \rightarrow \tilde{U}$ to a surface $\tilde{U}$ with a real analytic structure defined in terms of elements $\left(\beta, \tilde{F}_{\beta}\right)$ with $\beta \in f(U)$ by $\alpha=f^{-1}(\beta)$ and $\tilde{F}_{\beta}=F_{\alpha} \circ f^{-1}$. We again define a projection by $\pi:\left(\beta, \tilde{F}_{\beta}\right) \rightarrow \beta$.

We refer to a point $\hat{\zeta} \in \hat{U}$ to be over $\zeta$, if $p(\hat{\zeta})=\zeta$, and $\tilde{z} \in \tilde{U}$ to be over $z$ if $\pi(\tilde{z})=z$.

The harmonic mapping $f: U \rightarrow f(U)$ lifts to a mapping $\hat{f}: \hat{U} \rightarrow \tilde{U}$ which is $1-1$, onto, and satisfies the condition $\pi(\hat{f}(\hat{\zeta}))=f(p(\hat{\zeta}))$ for all $\zeta \in \hat{U}$. With these notations, we extend the meaning of (2.2). Thus

$$
\hat{\zeta} \rightarrow(\hat{f}(\hat{\zeta}), \operatorname{Re} \hat{F}(\hat{\zeta}))
$$

gives a parametric representation of a minimal surface in the sense that in a neighborhood of $\hat{\zeta} \in \hat{U} \backslash \mathcal{B}$ where $\mathcal{B}$ is the branch set, that is, the points above the zeros of $a$ of odd order, then (2.2) is the same as (2.3) computed in terms of local coordinates given by projection.

We may also define the surface nonparametrically on $\tilde{U} \backslash \tilde{\mathcal{B}}$, where $\tilde{\mathcal{B}}=\hat{f}(\mathcal{B})$, as follows. Let $D$ be an open disk in $f(U)$ such that $f^{-1}(D)$ contains no zeros of $a$ of odd multiplicity. Let $w=\varphi(x, y)$ be the nonparametric description of the minimal surface corresponding to (2.2), that is, for $\zeta \in f^{-1}(0)$ ( $c f$. [HS3, p. 87]),

$$
\begin{gathered}
x=\operatorname{Re} f(\zeta) \quad y=\operatorname{Im} f(\zeta), \\
\varphi(x, y)=\operatorname{Re} F(\zeta)
\end{gathered}
$$

Then, by continuation $\varphi$ lifts to a function $\tilde{\varphi}$ on $\tilde{U}$ which satisfies the minimal surface equation when computed in local coordinates given by projection off the branch 
set $\tilde{\mathcal{B}}$. We call $\tilde{\varphi}(\tilde{z})$ a height function corresponding to $f$. We define a conjugate height function $\tilde{\psi}(z)$ by solving locally

$$
\psi_{y}=\varphi_{x} / W, \psi_{x}=-\varphi_{y} / W \quad\left(W=\sqrt{1+\varphi_{x}^{2}+\varphi_{y}^{2}}\right)
$$

(cf. [JS, p. 326]), and lifting to $\tilde{U} \backslash \tilde{\mathcal{B}}$ as was done for $\varphi$. Let $\tilde{F}=\tilde{\varphi}+i \tilde{\psi}$. Then, as shown in [W], $\tilde{F}=\hat{F}+c$ is well defined on $\tilde{U} \backslash \tilde{B}$. Finally, we extend $\hat{F}$ and $\tilde{F}$ to $\hat{U}$ and $\tilde{U}$ respectively by continuity to the branch points.

A glossary of terminology is given schematically in Figure 1.

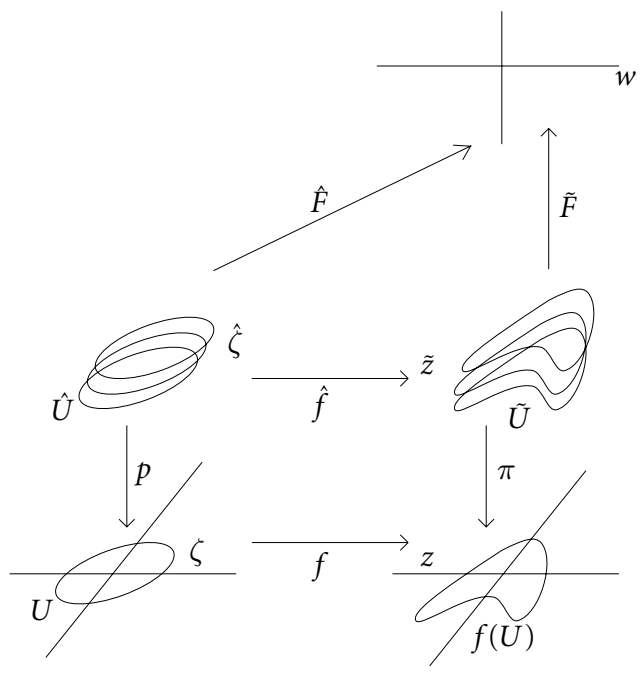

Figure 1

\section{The Comparison Principle for the Height Function}

In this section we point out that the comparison principle for solutions to the minimal surface equation

$$
\operatorname{div} \frac{\nabla u}{W}=0 \quad\left(W=\sqrt{1+|\nabla u|^{2}}\right)
$$

carries over to the height function corresponding to univalent harmonic mappings.

Let $f_{1}$ and $f_{2}$ be univalent harmonic mapping in $U$, and suppose $U_{0} \subseteq f_{1}(U) \cap$ $f_{2}(U) \neq \phi$, where $U_{0}$ is open, connected and bounded. Let $z_{0} \in U_{0}$ not be the image of a branch point of $f_{1}$ or $f_{2}$, and in a neighborhood $N$ of $z_{0}$ define $\varphi_{1}(x, y)$ and $\varphi_{2}(x, y)$ corresponding to $f_{1}$ and $f_{2}$ respectively, as is done for $\varphi(x, y)$ in $(2.4)$. Let 
$\Phi(x, y)=\varphi_{1}(x, y)-\varphi_{2}(x, y)$. We now consider continuations of $\Phi$ along all curves in $U_{0}$ emanating from $N$.

Theorem 1 Suppose that $\lim \sup \Phi \leq 0$ for any continuation along curves from $N$ in $U_{0}$, tending to points on $\partial U_{0}$. Then, the supremum $M$ of any continuation of $\Phi$ along curves in $U_{0}$ also satisfies $M \leq 0$.

Proof We may assume that $f_{1}$ and $f_{2}$ are univalent harmonic in $\bar{U}$ by considering $f_{1}(r z)$ and $f_{2}(r z)$ where $r<1$, and letting $r \rightarrow 1$. Thus we may assume the analytic dilatations for $f_{1}$ and $f_{2}$ have finitely many zeros, so their height functions have only a finite number of different function elements over each point. Let $z=z(t), 0 \leq t<1$, $z(0)=z_{0}$ be a curve along which the continuation of $\Phi$ tends to its supremum on some sequence. We assume, contrary to the theorem, that $M$ is positive. By hypothesis there exists $z^{*} \in U_{0}$ such that $z\left(t_{k}\right) \rightarrow z^{*}$ and $\Phi\left(z\left(t_{k}\right)\right) \rightarrow M$ for some sequence $t_{k} \rightarrow 1$. In order to analyze this, we consider the individual continuations of $\varphi_{1}$ and $\varphi_{2}$ along the curve and recall that $\varphi_{1}$ and $\varphi_{2}$ have only a finite number of function elements over each point of $U_{0}$. Thus, at least for some subsequence $t_{k_{n}} \rightarrow 1$, the continuation of $\Phi$ corresponds to fixed function elements of $\varphi_{1}$ and $\varphi_{2}$ over $z^{*}$. For these branches we then consider $\Phi=\varphi_{1}-\varphi_{2}$, and show that this determination of $\Phi$ cannot have a negative relative maximum at $z^{*}$. The theorem will then follow.

If $\varphi_{1}$ and $\varphi_{2}$ do not have a branch point at $z^{*}$, then the result follows from the usual comparison principle for solutions to $(2.1)$ (cf. [O, p. 91]).

If $\varphi_{1}$ or $\varphi_{2}$ do have a branch point at $z^{*}$, then we analyze $\Phi$ on a two sheeted surface $\tilde{D}$ over a disk $D=\left\{\left|z-z^{*}\right|<\rho\right.$ where we have assumed $\rho$ is small enough so that $z^{*}$ is the only branch point in $D$, and $\bar{D} \subseteq U_{0}$.

Following Nitsche [N], let $m$ and $\epsilon$ be positive constants, with $m$ large and $\epsilon$ small. In particular $\epsilon<\rho$. On $\tilde{D}$ define

$$
\tau= \begin{cases}m-\epsilon & \text { if } \varphi_{1}-\varphi_{2} \geq m \\ \Phi-\epsilon & \text { if } \epsilon<\varphi_{1}-\varphi_{2}<m \\ 0 & \text { if } \varphi_{1}-\varphi_{1} \leq \epsilon\end{cases}
$$

Let $\tilde{C}_{\epsilon}=\partial \tilde{D}_{\epsilon}$ be oriented positively, where $\tilde{D}_{\epsilon}$ is the subset of $\tilde{D}$ over $D \backslash\left\{\left|z-z^{*}\right|<\right.$ $\epsilon\}$. Then, using coordinates given by projection on $\tilde{D}$ and $W_{1}, W_{2}$ as defined in (3.1) for $\varphi_{1}$ and $\varphi_{2}$ in place of $u$, we have by (3.1) and the divergence theorem that

$$
\oint_{\tilde{C}_{\epsilon}} \tau\left(\frac{\nabla \varphi_{1}}{W_{1}} \cdot \nu-\frac{\nabla \varphi_{2}}{W_{2}} \cdot \nu\right) d s=\iint_{\tilde{D}_{\epsilon}} \nabla \tau \cdot\left(\frac{\nabla \varphi_{1}}{W_{1}}-\frac{\nabla \varphi_{2}}{W_{2}}\right) d A
$$

where $\nu$ is the outward unit normal.

It follows from (3.3) that

$$
8 \pi m \epsilon \geq \iint_{\tilde{D}_{\epsilon}} \nabla \tau \cdot\left(\frac{\nabla \varphi_{1}}{W_{1}}-\frac{\nabla \varphi_{2}}{W_{2}}\right) d A \geq 0
$$

Here we have also used the observation that with (3.2), the integrand is nonnegative. As $\epsilon \rightarrow 0$ the sets $\tilde{D}_{\epsilon}$ expand and we obtain the fact that $\nabla \varphi_{1}=\nabla \varphi_{2}$ in the set $\{0<$ 
$\left.\varphi_{1}-\varphi_{2}<m\right\}$. Thus $\varphi_{1} \equiv \varphi_{2}+$ constant in any component of $\tilde{D}$ where $\varphi_{1}>\varphi_{2}$. Since $\varphi_{1}$ and $\varphi_{2}$ are real analytic off the branch set, we obtain a contradiction unless the set where $\varphi_{1}>\varphi_{2}$ is empty.

\section{Combinatorial Properties of the Conjugate Height Function}

In this section we shall collect the relevant facts which enable us to give combinatorial arguments using the conjugate height functions.

Let $\tilde{\psi}$ be a conjugate height function for a univalent harmonic mapping $f$ in $U$. We assume in Theorems A, B, C below that $f(U)$ is bounded, and its analytic dilatation has finitely many zeros of odd order so that $\tilde{U}$ is finite sheeted. In the present context, [JS, p. 327] gives

Theorem A Let $\tilde{C}$ be a simple piecewise smooth curve in the closure of $\tilde{U}$. Then, using the coordinates given by projection,

$$
\left|\int_{\tilde{C}} d \tilde{\psi}\right| \leq \operatorname{length}(\tilde{C})
$$

with strict inequality if any portion of $\tilde{C}$ lies over points in $\tilde{U}$.

Moreover [JS, Lemma 2], we have

Theorem B If $\tilde{C}$ is a simple, piecewise smooth closed curve in the closure of $\tilde{U}$, then $\int_{\tilde{C}} d \tilde{\psi}=0$.

The companion to Theorems A and B is provided by Lemma 4 of [JS]. Again paraphrasing, in the current setting we have

Theorem $C$ Suppose that $\partial f(U)$ contains a line segment $T$, and $\tilde{T}$ is a segment over $T$ in $\tilde{U}$. If $\tilde{T}$ is oriented so that the right hand normal to $\tilde{T}$ is the outer normal to $\tilde{U}$, and $\tilde{\varphi}=+\infty$ on $\tilde{T}$, then in the coordinates given by projection,

$$
\int_{\tilde{T}} d \tilde{\psi}=\operatorname{length}(T) .
$$

The value of Theorems A, B, and C when applied to Poisson integrals of step functions stem from [W, Theorem 2]

Theorem $\boldsymbol{D}$ Let $\mathcal{P}$ be a polygon having vertices $c_{1}, \ldots, c_{n}$ given cyclically on $\partial \mathcal{P}$, and ordered by a positive orientation on $\partial \mathcal{P}$. Let $f$ be a univalent harmonic mapping of $U$ such that $f$ is the Poisson integral of a step function having the ordered sequence $c_{1}, \ldots, c_{n}$ as its values. Then the analytic dilatation $a(\zeta)$ of $f$ is a finite Blaschke product of order at most $n-2, f(U)=\mathcal{P}$, with equality if $\mathcal{P}$ is convex. If $\tilde{\varphi}$ is a height function for $f$, then $\tilde{\varphi}$ tends to $+\infty$ or $-\infty$ at points over the open segments making up the sides of $\mathcal{P}$. At any vertex $c_{j}$ of $\mathcal{P}$ at which the interior angle is less than $\pi$, then $+\infty$ and $-\infty$ alternate on adjacent sides having $c_{j}$ as the common vertex.

The proofs of Theorems A, B, and C are just as given in [JS]. The statement of Theorem D differs in the last sentence in [W, Theorem 2], but the statement given above is actually what is proved there. 


\section{Poisson Integrals of Step Functions}

Throughout this section we use the notations of Theorem D. The classical Scherk surface arises from taking $c_{1}=0, c_{2}=1, c_{3}=1+i, c_{4}=i$ as vertices, and with the Poisson integral $f$ taking those values on respective intervals of equal length, and ordered positively around $\partial U$. Then $a(\zeta)=c \zeta^{2}$, and the height function can be taken as a saddle with heights $\pm \infty$ alternately over the sides of the square. As forecast by Theorem $\mathrm{D}, a(\zeta)$ has two zeros (counting multiplicity). In order to motivate the general phenomenon, suppose we extend the top and bottom sides of the square to make a rectangle $R$, and stretch or shrink the corresponding intervals for the new $f$ in $\partial U$ in any fashion. Still, since $R$ is convex, the analytic dilatation will have two zeros $\zeta_{1}, \zeta_{2}$. As we shall see in the proof of Theorem 2, the images $z_{1}=f\left(\zeta_{1}\right), z_{2}=f\left(\zeta_{2}\right)$ of these points lie cannot both be to the left or right of the center of $R$, regardless of the relative sizes of the intervals in $\partial U$ corresponding to the vertices of $R$.

In general, if $f$ comes from the Poisson integral of a step function mapping $U$ onto a polygon $\mathcal{P}$ with the values of the step function being vertices $c_{1}, \ldots, c_{n}$, then its analytic dilatation $a(\zeta)$ has at most $n-2$ zeros in $U$. Theorem 2 shows that if we have some knowledge of $\partial \mathcal{P}$, we can say more.

Theorem 2 With $f, a, \mathcal{P}$, and $c_{1}, \ldots, c_{n}$ as above, suppose that the interior angles at $c_{j}$ and $c_{j+1}$ are less than $\pi$. Let $d_{j}, d_{j+1}$ be points on the segments $c_{j-1} c_{j}$ and $c_{j+1} c_{j+2}$ respectively, and assume that the open quadrilateral $\mathcal{P}_{j}$ with vertices $d_{j}, c_{j}, c_{j+1}, d_{j+1}$ is contained in $f(U)$. If length $\left(d_{j} d_{j+1}\right)+$ length $\left(c_{j} c_{j+1}\right)<$ length $\left(d_{j} c_{j}\right)+$ length $\left(c_{j+1} d_{j+1}\right)$, then $\mathcal{P}_{j}$ contains the image of at least one zero of $a(\zeta)$ of odd order. Thus, in particular, if $\mathcal{P}$ has $k$ disjoint such quadrilaterals, then $a(\zeta)$ has at least $k$ zeros.

Proof Suppose that $\mathcal{P}_{j}$ were such a quadrilateral without a branch point. Then there would exist a single valued branch of the height function $\tilde{\varphi}$ over $\mathcal{P}_{j}$ whose values over $d_{j} c_{j}, c_{j} c_{j+1}, c_{j+1} d_{j+1}$ would alternate $\pm \infty$ by Theorem D. Let $\tilde{\gamma}$ be the boundary of a quadrilateral $\tilde{\mathcal{P}}_{j}$ over $\mathcal{P}_{j}$ in $\tilde{U}$, oriented positively. We have from Theorem B that

$$
\int_{\tilde{\gamma}} d \tilde{\psi}=0
$$

By the alternation of signs, if $\tilde{\gamma}_{1}$ is the edge over $d_{j} c_{j}$, and $\tilde{\gamma}_{3}$ is over $c_{j+1} d_{j+1}$, then by Theorem C,

$$
\left|\int_{\tilde{\gamma}_{1}} d \tilde{\psi}+\int_{\tilde{\gamma}_{3}} d \tilde{\psi}\right|=\text { length }\left(d_{j} c_{j}\right)+\operatorname{length}\left(c_{j+1} d_{j+1}\right) .
$$

From (5.1) and (5.2) we then obtain

$$
\text { length }\left(d_{j} c_{j}\right)+\text { length }\left(c_{j+1} d_{j+1}\right) \leq\left|\int_{\tilde{\gamma}_{2}} d \tilde{\psi}\right|+\left|\int_{\tilde{\gamma}_{4}} d \tilde{\psi}\right|
$$

where $\tilde{\gamma}_{2}$ is over $c_{j} c_{j+1}$, and $\tilde{\gamma}_{4}$ is over $d_{j} d_{j+1}$. 
Again, by Theorem C,

$$
\left|\int_{\tilde{\gamma}_{2}} d \tilde{\psi}\right|=\operatorname{length}\left(c_{j} c_{j+1}\right)
$$

and by Theorem A,

$$
\left|\int_{\tilde{\gamma}_{4}} d \tilde{\psi}\right| \leq \text { length }\left(d_{j} d_{j+1}\right) \text {. }
$$

Combining (5.3)-(5.5), we contradict the hypothesis of the theorem. Thus, $\mathcal{P}_{j}$ must contain the image of at least one zero of odd order of $a(\zeta)$.

\section{References}

[C] G. Choquet, Sur un type de transformation analytique généralisant la représentation conforme et définie an moyen de fonctions harmoniques. Bull. Sci. Math. 69(1945), 156-165.

[CS-S] J. Clunie and T. Sheil-Small, Harmonic univalent functions. Ann. Acad. Sci. Fenn. Ser. A I Math. 9(1984), 3-25.

[HS] W. Hengartner and G. Schober, On the boundary behavior of orientation-preserving harmonic mappings. Complex Variables 5(1986), 197-208.

[JS] H. Jenkins and J. Serrin, Variational problems of minimal surface type II. Boundary value problems for the minimal surface equation. Arch. Rat. Mech. Anal. 21(1965/66), 321-342.

[K] H. Kneser, Lösung der Aufgabe 41. Jahresber. Deutsch. Math.-Verein. 35(1925), 123-4.

[N] J. C. C. Nitsche, Über ein verallgemeinertes Dirichletsches Problem für die Minimalflächengleichung und hebbare Unstetigkeiten ihrer Lösungen. Math. Ann. 158(1965), 203-214.

[O] R. Osserman, A Survey of Minimal Surfaces. Dover, 1986.

[S] G. Springer, Introduction to Riemann Surfaces. Addison-Wesley, 1957.

[S-S] T. Sheil-Small, On the Fourier series of a step function. Michigan Math. J. 36(1989), 459-475.

[W] A. Weitsman, On Univalent Harmonic Mappings and Minimal Surfaces. Pacific Math. J. 192(2000), $191-200$.

Department of Mathematics

Purdue University

West Lafayette, Inidiana 47907

U.S.A. 